\title{
Family Studies on Ocular Refraction and Its Components
}

\author{
ARNOLD SORSBY*, G. A. LEARY, and G. R. FRASER \\ From the Ophthalmological Research Department, Royal Eye Hospital and Royal College of Surgeons
}

A study on 78 pairs of uniovular twins contrasted with 40 pairs of binovular twins and 48 pairs of unrelated subjects has established that refraction as a whole and its major individual componentsthe axial length and the powers of the cornea and lens-are genetically determined both for emmetropia and the various types of refractive errors, including those of a high degree (Sorsby, Sheridan, and Leary, 1962; Sorsby and Fraser, 1964). The coefficients of correlation in these different refractive states for refraction and its components approached unity in the uniovular twins, 0.5 in the binovular twins, and zero in the unrelated controls. It is likely that more than one mode of inheritance comes into play, and family studies have been undertaken to evaluate this aspect.

\section{Material and Methods}

Twenty-eight families with a total of 106 subjects were studied. To eliminate disturbances arising from senescent changes in the parents, and from uncompleted growth in the offspring, only families in which the parents were under 63 years and the offspring studied were 16 years and over were considered. Any family in which either of the parents showed a pathological eye trait (other than an unusual refraction) was excluded. In all other respects the families were an unselected and probably a representative sample of the general population. The detailed findings are recorded in the Appendix.

In addition to these families, the records were also studied of a group of $2 \mathrm{I}$ families selected for unusual refraction in either one of the parents or one of the offspring. This group has been reported in a previous study (Sorsby, Benjamin, Davey, Sheridan, and Tanner, 1957), and a further statistical assessment is attempted here.

The methods employed have been recorded in the two previous studies and are discussed more fully elsewhere (Sorsby, Benjamin, and Sheridan, 1961).

Received July 6, 1966.

*In receipt of a grant for the Wernher Trustees, Medical Research Council.

\section{Analysis}

The coefficients of correlation in the 28 unselected families are set out in Table I. The over-all pattern is clear enough: in contrast to the lack of correlation between the parents, there is a substantial degree of correlation for parent/child and for mid-parent/offspring coefficients, though these coefficients fall short of the values of 0.50 and 0.71 expected under the hypothesis of the additive effects of a number of genes without dominance. The coefficients for the relatively small number of sibs available approach the expected value of 0.5 for refraction as a whole and for axial length and the power of the lens.

\section{TABLE I}

COEFFICIENTS OF CORRELATION FOR REFRACTION AND ITS COMPONENTS IN 28 UNSELECTED FAMILIES

\begin{tabular}{l|c|c|c|c}
\hline & $\begin{array}{c}\text { Father/ } \\
\text { Mother } \\
\text { 28 Pairs }\end{array}$ & $\begin{array}{c}\text { Parent/ } \\
\text { Child } \\
\text { roo }\end{array}$ & $\begin{array}{c}\text { Mid- } \\
\text { parent/ } \\
\text { Offspring } \\
\text { 50 }\end{array}$ & $\begin{array}{c}\text { Sib/Sib } \\
24 / 22\end{array}$ \\
\hline Refraction & -0.119 & 0.227 & 0.348 & 0.358 \\
Axial length & 0.108 & 0.314 & 0.434 & 0.492 \\
Corneal power & 0.138 & 0.378 & 0.542 & 0.093 \\
Lens power & -0.288 & 0.155 & 0.278 & 0.553 \\
$\begin{array}{l}\text { Anterior chamber } \\
\text { depth }\end{array}$ & -0.098 & 0.265 & 0.388 & 0.019 \\
\hline
\end{tabular}

In the previously reported families, selected for unusual refraction in the parents or offspring, the coefficients of correlation for the retraction and its components set out in Table II (recalculated on the same basis as the material recorded here) show that with a high degree of correlation for refraction and axial length for the two parents, there is a high degree of correlation for refraction and all components except axial length between parents and children. In this series the small number of sibs precludes any worth-while analysis of relevant coefficients. 
TABLE II

COEFFICIENTS OF CORRELATION FOR REFRACTION AND ITS COMPONENTS IN $2 I$ FAMILIES WITH UNUSUAL REFRACTION IN EITHER THE PARENTS OR THE OFFSPRING

\begin{tabular}{l|c|c|c|c}
\hline & $\begin{array}{c}\text { Father/ } \\
\text { Mother } \\
\text { 21 pairs }\end{array}$ & $\begin{array}{c}\text { Parent/ } \\
\text { Child } \\
56\end{array}$ & $\begin{array}{c}\text { Mid- } \\
\text { Parent/ } \\
\text { Offspring } \\
28\end{array}$ & $\begin{array}{c}\text { Sib/Sib } \\
7\end{array}$ \\
\hline Refraction & 0.339 & 0.285 & 0.342 & -0.104 \\
Axial length & 0.321 & 0.062 & 0.076 & -0.122 \\
Corneal power & -0.039 & 0.271 & 0.401 & -0.289 \\
Lens power & -0.099 & 0.416 & 0.580 & 0.349 \\
Anterior chamber & 0.1 I I & 0.323 & 0.412 & 0.615 \\
\hline
\end{tabular}

Note: In this Table the second of each of two pairs of identical twins (families 2 and 8 ) have been omitted.

\section{Discussion}

The 28 unselected families included six families in which one or both parents had a refractive error outside the range of +4.00 to $-4.00 \mathrm{D}$. With a larger number of families, these two groups of refraction might with advantage have been considered separately, but even with the present small numbers the only unexpected findings are the relatively low coefficients for the cornea and the anterior chamber in sibs, and for the lens in parent/ child relationship. The available evidence, therefore, points to the strong probability that with larger numbers inheritance of refraction and its components in the general population is likely to follow the pattern set by a number of genes with additive effect.

The findings for the selected cases suggest that this also applies to the different components of refraction, other than the axial length which has characteristic features of its own. The study on twins has shown axial length to be genetically determined, while the high correlation coefficients for the cornea and lens seen in the present stud emphasize that the compensating mechanism of reduction in the powers of the cornea and lens aro effective for variations in axial length within the range seen in emmetropia but not beyond. It likely that-in contrast to the other components of refraction including axial lengths within the emmetropic range-abnormally long or short axim lengths are determined monofactorially, and it if fairly certain that there is more than one mode of inheritance of such abnormal axes. These are possibilities that need further exploration.

\section{Summary}

Twenty-eight unselected families with a total of I06 subjects were studied for ocular refraction and its components. In contrast to the lack of correlas tion between the parents, there was a substantial degree of correlation for parent/child and for mide parent/offspring coefficients.

In 2 I previously reported families selected fơ unusual refraction and recalculated on the sam basis as the material recorded here, there was $\mathbb{Q}$ high degree of correlation between parents an children for refraction and all components excep axial length.

Our thanks are due to Miss E. M. Gower and Mis Joan Richards for assistance in this work.

\section{REFERENCES}

Sorsby, A., Beniamin, B., Davey, J. B., Sheridan, M., and Tanne J. M. (1957). Emmetropia and its aberrations. Spec. Rep. Ser. med. Res. Coun. (Lond.), No. 293.

,-- and Sheridan, M. (196I). Refraction and its componenc during the growth of the eye. ibid., No. 301 .

- , and Fraser, G. R. (1964). Statistical note on the components ocular refraction in twins. F. med. Genet., $1,47$.

- - Sheridan, M., and Leary, G. A. (I962). Refraction and it components in twins. Spec. Rep. Ser. med. Res. Coun. (Lond No. 303 .

\section{Appendix}

Refraction and its components in 28 families with a total of 106 subjects

\begin{tabular}{|c|c|c|c|c|c|c|c|c|}
\hline $\begin{array}{c}\text { Family } \\
\text { No. }\end{array}$ & Member & Age (yr.) & $\begin{array}{c}\text { Ocular Refraction } \\
\text { in Vertical } \\
\text { Meridian } \\
\text { (D) }\end{array}$ & $\begin{array}{l}\text { Corneal } \\
\text { Power in } \\
\text { Vertical } \\
\text { Meridian } \\
\text { (D) }\end{array}$ & $\begin{array}{l}\text { Anterior } \\
\text { Chamber } \\
\text { Depth } \\
(\mathrm{mm} .)\end{array}$ & $\begin{array}{l}\text { Lens } \\
\text { Equivalent } \\
\text { Power } \\
\text { (D) }\end{array}$ & $\begin{array}{c}\text { Lens } \\
\text { Power at } \\
\text { Corneal Vertex } \\
\text { (D) }\end{array}$ & $\begin{array}{r}\text { Axial } \\
\text { Length } \\
\text { (mm.) }\end{array}$ \\
\hline I & $\begin{array}{l}\text { Father } \\
\text { Mother } \\
\text { Son } \\
\text { Daughter } \\
\text { Daughter }\end{array}$ & $\begin{array}{l}56 \\
55 \\
25 \\
24 \\
16\end{array}$ & $\begin{array}{l}R-I .48 \\
\mathrm{~L}-\mathrm{I} \cdot 72 \\
\mathrm{R}+\mathrm{I} \cdot 26 \\
\mathrm{~L}+\mathrm{I} \cdot 56 \\
\mathrm{R}-\mathbf{3} .59 \\
\mathrm{~L}-\mathbf{4} .27 \\
\mathrm{R}+0.25 \\
\mathrm{~L}+0.50 \\
\mathrm{R}+0.26 \\
\mathrm{~L}+0.50\end{array}$ & $\begin{array}{l}43 \cdot 7 \\
44 \cdot 1 \\
4 I \cdot 4 \\
4 I \cdot 3 \\
4 I \cdot 7 \\
4 I \cdot 7 \\
42 \cdot 3 \\
42 \cdot 1 \\
4 I \cdot 8 \\
4 I \cdot 7\end{array}$ & $\begin{array}{l}3 \cdot 2 \\
3 \cdot 5 \\
3 \cdot 5 \\
3 \cdot 5 \\
3 \cdot 6 \\
3 \cdot 6 \\
3 \cdot 9 \\
3 \cdot 7 \\
3.6 \\
3 \cdot 6\end{array}$ & $\begin{array}{l}21 \cdot 7 \\
22.6 \\
19.5 \\
19.1 \\
21.8 \\
22.8 \\
19.7 \\
19.2 \\
20.8 \\
20.8\end{array}$ & $\begin{array}{l}12 \cdot 8 \\
12 \cdot 9 \\
111 \cdot 9 \\
111 \cdot 5 \\
13.7 \\
14.4 \\
111.6 \\
111 \cdot 5 \\
12.8 \\
12.7\end{array}$ & $\begin{array}{l}24 \cdot 2 \\
24 \cdot 1 \\
24 \cdot 5 \\
24 \cdot 5 \\
25 \cdot 7 \\
25 \cdot 7 \\
24 \cdot 6 \\
24 \cdot 7 \\
24 \cdot 3 \\
24 \cdot 3\end{array}$ \\
\hline
\end{tabular}




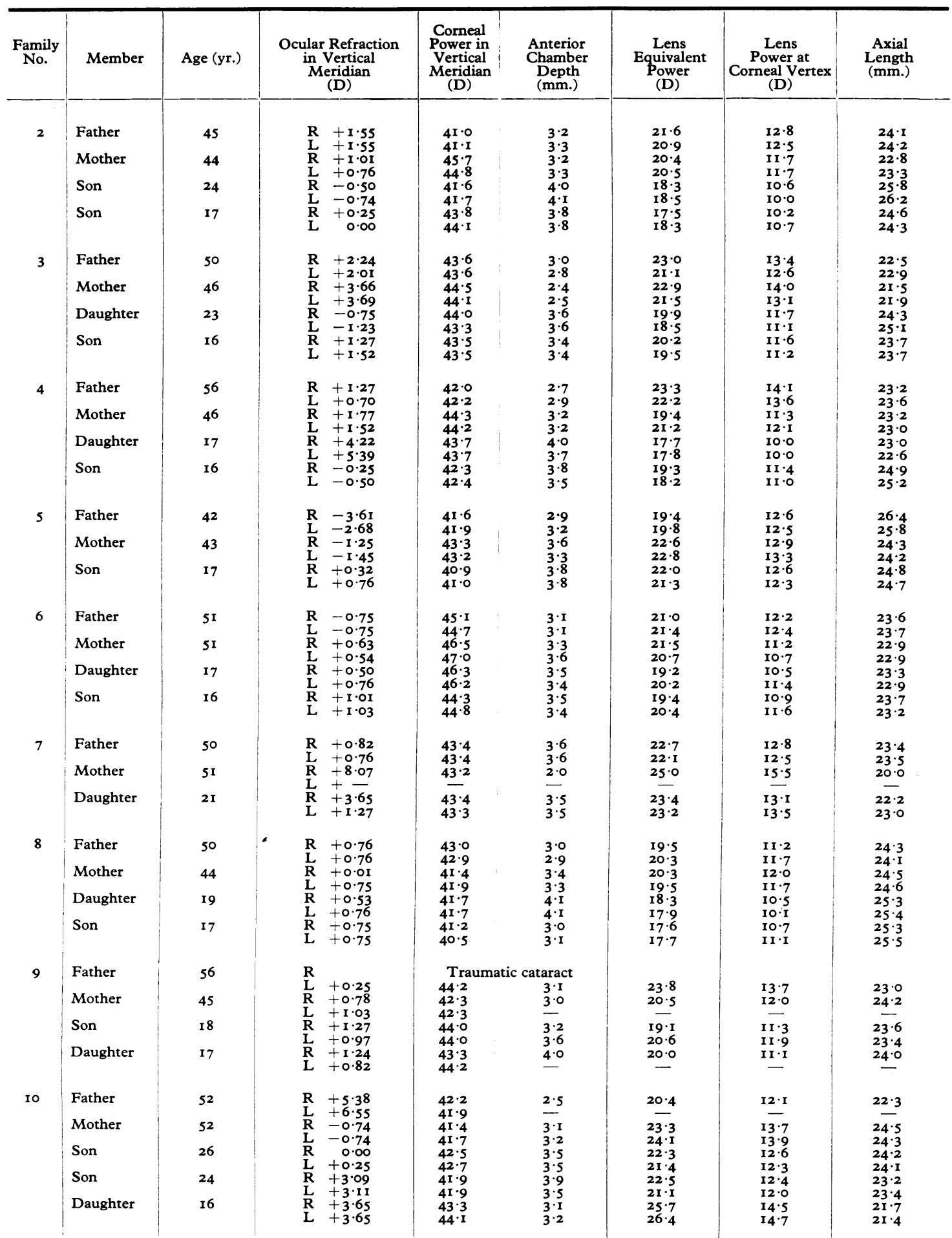




\begin{tabular}{|c|c|c|c|c|c|c|c|c|}
\hline $\begin{array}{l}\text { Family } \\
\text { No. }\end{array}$ & Member & Age (yr.) & $\begin{array}{l}\text { Ocular Refraction } \\
\text { in Vertical } \\
\text { Meridian } \\
\text { (D) }\end{array}$ & $\begin{array}{l}\text { Corneal } \\
\text { Power in } \\
\text { Vertical } \\
\text { Meridian } \\
\text { (D) }\end{array}$ & $\begin{array}{c}\text { Anterior } \\
\text { Chamber } \\
\text { Depth } \\
\text { (mm.) }\end{array}$ & $\begin{array}{c}\begin{array}{c}\text { Lens } \\
\text { Equivalent } \\
\text { Power } \\
\text { (D) }\end{array} \\
\end{array}$ & $\begin{array}{c}\text { Lens } \\
\text { Power at } \\
\text { Corneal Vertex } \\
\text { (D) }\end{array}$ & $\begin{array}{l}\text { Axial } \\
\text { Length } \\
(\mathrm{mm} .)\end{array}$ \\
\hline II & \begin{tabular}{|l} 
Father \\
Mother \\
Son \\
Daughter
\end{tabular} & $\begin{array}{l}42 \\
39 \\
19 \\
17\end{array}$ & 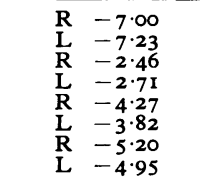 & $\begin{array}{l}43 \cdot 9 \\
43 \cdot 9 \\
42 \cdot 6 \\
42 \cdot 7 \\
42 \cdot 8 \\
42 \cdot 9 \\
43 \cdot 2 \\
43 \cdot 2\end{array}$ & $\begin{array}{l}\overline{3 \cdot 6} \\
\frac{3 \cdot 8}{3 \cdot 8} \\
4 \cdot 0 \\
3 \cdot 3 \\
3 \cdot 8\end{array}$ & $\begin{array}{l}- \\
23 \cdot 9 \\
22 \cdot 3 \\
20 \cdot 5 \\
17 \cdot 9 \\
21 \cdot 4\end{array}$ & $\begin{array}{l}15.1 \\
15- \\
13 \cdot 4 \\
12 \cdot .1 \\
11.3 \\
12 \cdot 8\end{array}$ & $\begin{array}{l}- \\
25 \cdot 8 \\
25 \cdot 0 \\
26 \cdot 3 \\
26 \cdot 5 \\
26 \cdot 1\end{array}$ \\
\hline 12 & $\begin{array}{l}\text { Father } \\
\text { Mother } \\
\text { Son }\end{array}$ & $\begin{array}{l}54 \\
55 \\
18\end{array}$ & $\begin{array}{lr}\mathrm{R} & +0.25 \\
\mathrm{~L} & +0.25 \\
\mathrm{R} & +0.56 \\
\mathrm{~L} & +\mathrm{I} .0 \mathrm{I} \\
\mathrm{R} & -0.47 \\
\mathrm{~L} & 0.00\end{array}$ & $\begin{array}{l}43 \cdot 7 \\
43 \cdot 7 \\
4 \cdot I \\
4 \cdot I \\
43 \cdot I \\
42 \cdot 7\end{array}$ & $\begin{array}{l}2 \cdot 7 \\
2 \cdot 6 \\
3 \cdot 0 \\
2 \cdot 8 \\
3 \cdot 2 \\
3 \cdot 4\end{array}$ & $\begin{array}{l}21 \cdot 6 \\
22 \cdot 2 \\
24 \cdot 0 \\
23 \cdot 1 \\
18 \cdot 5 \\
18 \cdot 9\end{array}$ & $\begin{array}{l}12.7 \\
13.4 \\
14.2 \\
13.8 \\
11.5 \\
11.5\end{array}$ & $\begin{array}{l}23 \cdot 5 \\
23 \cdot 3 \\
22 \cdot 7 \\
22 \cdot 6 \\
24 \cdot 7 \\
24 \cdot 6\end{array}$ \\
\hline 13 & $\begin{array}{l}\text { Father } \\
\text { Mother } \\
\text { Son } \\
\text { Son }\end{array}$ & $\begin{array}{l}54 \\
42 \\
21 \\
19\end{array}$ & 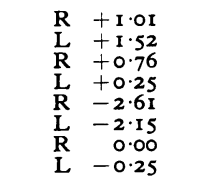 & $\begin{array}{l}42 \cdot 8 \\
42 \cdot 3 \\
44 \cdot 0 \\
43 \cdot 3 \\
44 \cdot 2 \\
44 \cdot 0 \\
41 \cdot 0 \\
40 \cdot 5\end{array}$ & $\begin{array}{l}2.5 \\
2.6 \\
3.8 \\
3.5 \\
3.4 \\
3.2 \\
4.0 \\
3.9\end{array}$ & $\begin{array}{l}26 \cdot 1 \\
24 \cdot 2 \\
20 \cdot 1 \\
19 \cdot 8 \\
21 \cdot 1 \\
21.7 \\
18 \cdot 5 \\
18 \cdot 7\end{array}$ & $\begin{array}{l}15.3 \\
14.0 \\
11.3 \\
11.5 \\
12.8 \\
13.2 \\
10.7 \\
11.1\end{array}$ & $\begin{array}{l}22 \cdot 6 \\
23 \cdot 1 \\
23 \cdot 8 \\
24 \cdot 2 \\
24 \cdot 5 \\
24 \cdot 2 \\
25 \cdot 8 \\
26 \cdot 0\end{array}$ \\
\hline 14 & $\begin{array}{l}\text { Father } \\
\text { Mother } \\
\text { Son } \\
\text { Daughter }\end{array}$ & $\left.\begin{array}{l}42 \\
41 \\
16 \\
16\end{array}\right\}$ Twin & $\begin{array}{l}\mathrm{R}+\mathrm{I} \cdot 29 \\
\mathrm{~L}+\mathrm{I} \cdot 27 \\
\mathrm{R}+\mathrm{I} \cdot 0 \mathrm{I} \\
\mathrm{L}+0.47 \\
\mathrm{R}+0.76 \\
\mathrm{~L}+0.77 \\
\mathrm{R}+0.27 \\
\mathrm{~L}+0.76\end{array}$ & $\begin{array}{l}44 \cdot 3 \\
44 \cdot 6 \\
43 \cdot 6 \\
44 \cdot 2 \\
43 \cdot 6 \\
43 \cdot 9 \\
44 \cdot 3 \\
44 \cdot 2\end{array}$ & $\begin{array}{l}2 \cdot 8 \\
2 \cdot 6 \\
3 \cdot 0 \\
3 \cdot 4 \\
3 \cdot 3 \\
3 \cdot 2 \\
\end{array}$ & $\begin{array}{l}25 \cdot 0 \\
23 \cdot 9 \\
19 \cdot 0 \\
20 \cdot 7 \\
20 \cdot 5 \\
21 \cdot 4\end{array}$ & $\begin{array}{l}14 \cdot 4 \\
14 \cdot 1 \\
11.2 \\
11.8 \\
11.9 \\
12.6\end{array}$ & $\begin{array}{l}22.2 \\
22.2 \\
23.9 \\
23.8 \\
23.6 \\
23.3\end{array}$ \\
\hline 15 & $\begin{array}{l}\text { Father } \\
\text { Mother } \\
\text { Daughter } \\
\text { Daughter }\end{array}$ & $\begin{array}{l}55 \\
59 \\
26 \\
16\end{array}$ & $\begin{array}{l}\mathrm{R}+2.56 \\
\mathrm{~L}+3.36 \\
\mathrm{R}-\mathrm{I} 5.94 \\
\mathrm{~L}-19.70 \\
\mathrm{R}+0.69 \\
\mathrm{~L}+\mathrm{I} .54 \\
\mathrm{R}+0.02 \\
\mathrm{~L}+0.02\end{array}$ & $\begin{array}{l}4 \mathrm{r} \cdot 2 \\
41 \cdot 0 \\
47 \cdot 3 \\
47 \cdot 6 \\
41 \cdot 9 \\
41 \cdot 9 \\
43 \cdot 3 \\
43 \cdot 0\end{array}$ & $\begin{array}{l}2 \cdot 6 \\
2 \cdot 7 \\
3 \cdot 3 \\
3 \cdot 1 \\
3 \cdot 3 \\
3 \cdot 1 \\
3 \cdot 3 \\
3 \cdot 4\end{array}$ & $\begin{array}{l}20 \cdot 5 \\
20.2 \\
24.4 \\
27.8 \\
20.7 \\
19 \cdot 8 \\
19.6 \\
20.5\end{array}$ & $\begin{array}{l}13.0 \\
12.8 \\
16.0 \\
18.8 \\
12.6 \\
12.2 \\
12.0 \\
12.4\end{array}$ & $\begin{array}{l}23 \cdot 5 \\
23 \cdot 3 \\
28 \cdot 1 \\
28 \cdot 6 \\
24 \cdot 2 \\
24 \cdot 0 \\
24 \cdot 1 \\
24 \cdot 1\end{array}$ \\
\hline 16 & $\begin{array}{l}\text { Father } \\
\text { Mother } \\
\text { Daughter } \\
\text { Daughter }\end{array}$ & $\begin{array}{l}44 \\
43 \\
19 \\
16\end{array}$ & 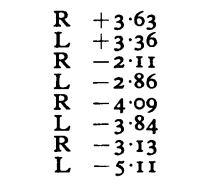 & $\begin{array}{l}42 \cdot 1 \\
41 \cdot 8 \\
43 \cdot 4 \\
43 \cdot 6 \\
44 \cdot 4 \\
45 \cdot 5 \\
41 \cdot 5 \\
42 \cdot 5\end{array}$ & $\begin{array}{l}3 \cdot 4 \\
3 \cdot 1 \\
3 \cdot 3 \\
3.8 \\
3.7 \\
4.0 \\
3.9\end{array}$ & $\begin{array}{l}21 \cdot 7 \\
18 \cdot 6 \\
19 \cdot 0 \\
17 \cdot 3 \\
18 \cdot 3 \\
19 \cdot 4 \\
20 \cdot 0\end{array}$ & $\begin{array}{l}\text { II.9 } \\
\text { II. } \\
\text { II. } \\
\text { IO.5 } \\
\text { II. } \\
\text { II. } \\
12.6\end{array}$ & $\begin{array}{l}23 \cdot 2 \\
25 \cdot 2 \\
25 \cdot 5 \\
26 \cdot 2 \\
25 \cdot 4 \\
26 \cdot 5 \\
26 \cdot 7\end{array}$ \\
\hline I7 & $\begin{array}{l}\text { Father } \\
\text { Mother } \\
\text { Son }\end{array}$ & $\begin{array}{l}43 \\
49 \\
16\end{array}$ & $\begin{array}{l}\mathrm{R}+\mathrm{I} \cdot 52 \\
\mathrm{~L}+\mathrm{I} \cdot 52 \\
\mathrm{R}+\mathrm{I} .53 \\
\mathrm{~L}+\mathrm{I} \cdot 79 \\
\mathrm{R}+\mathrm{I} \cdot 0 \mathrm{I} \\
\mathrm{L}+\mathrm{I} .0 \mathrm{I}\end{array}$ & $\begin{array}{l}42 \cdot 3 \\
42 \cdot 0 \\
43 \cdot 5 \\
43 \cdot 4 \\
44 \cdot 2 \\
44 \cdot 2\end{array}$ & $\begin{array}{l}3 \cdot 2 \\
3 \cdot 2 \\
3 \cdot 0 \\
3 \cdot 9 \\
3 \cdot 5\end{array}$ & $\begin{array}{l}22 \cdot 6 \\
23 \cdot 2 \\
21 \cdot 5 \\
22 \cdot 2 \\
22 \cdot 7\end{array}$ & $\begin{array}{l}12.8 \\
13.0 \\
12.6 \\
12.4 \\
13.0\end{array}$ & $\begin{array}{l}23 \cdot 5 \\
\frac{23 \cdot 0}{23 \cdot 1} \\
23 \cdot 2 \\
22 \cdot 9\end{array}$ \\
\hline 18 & $\begin{array}{l}\text { Father } \\
\text { Mother } \\
\text { Daughter } \\
\text { Daughter }\end{array}$ & $\begin{array}{l}62 \\
53 \\
20 \\
18\end{array}$ & 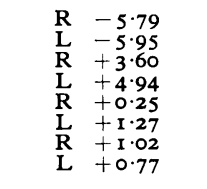 & $\begin{array}{l}45 \cdot 3 \\
45 \cdot 3 \\
46 \cdot 0 \\
45 \cdot 9 \\
46 \cdot 2 \\
46 \cdot 2 \\
41 \cdot 5 \\
41 \cdot 5\end{array}$ & $\begin{array}{l}2 \cdot 5 \\
2 \cdot 6 \\
2 \cdot 6 \\
2 \cdot 7 \\
3 \cdot 5 \\
3 \cdot 5 \\
3 \cdot 1 \\
3 \cdot 1\end{array}$ & $\begin{array}{l}22 \cdot 4 \\
22 \cdot 1 \\
21 \cdot 1 \\
19 \cdot 7 \\
19 \cdot .0 \\
18.8 \\
20 \cdot 3 \\
20.2\end{array}$ & $\begin{array}{l}14 \cdot 2 \\
13 \cdot 4 \\
12 \cdot 1 \\
11 \cdot 0 \\
11 \cdot 1 \\
10.9 \\
12 \cdot 6 \\
12 \cdot 6\end{array}$ & $\begin{array}{l}24 \cdot 8 \\
25 \cdot 3 \\
21 \cdot 6 \\
21 \cdot 5 \\
23 \cdot 2 \\
22 \cdot 8 \\
24 \cdot 2 \\
24 \cdot 3\end{array}$ \\
\hline 19 & $\begin{array}{l}\text { Father } \\
\text { Mother } \\
\text { Son }\end{array}$ & $\begin{array}{l}48 \\
48 \\
21\end{array}$ & $\begin{array}{ll}\mathrm{R} & +0.50 \\
\mathrm{~L} & +0.50 \\
\mathrm{R} & -0.50 \\
\mathrm{~L} & -\mathrm{I} .48 \\
\mathrm{R} & +0.6 \mathrm{I} \\
\mathrm{L} & +0.25\end{array}$ & $\begin{array}{l}42 \cdot 1 \\
41 \cdot 7 \\
44 \cdot 2 \\
43 \cdot 7 \\
40 \cdot 2 \\
40 \cdot 7\end{array}$ & $\begin{array}{l}3 \cdot 6 \\
3.4 \\
3 \cdot 2 \\
3.1 \\
3 \cdot 6 \\
3 \cdot 6\end{array}$ & $\begin{array}{l}19.8 \\
19.9 \\
22.8 \\
21.3 \\
19.6 \\
19 \cdot 4\end{array}$ & $\begin{array}{l}11 \cdot 5 \\
11 \cdot 7 \\
13 \cdot 1 \\
12 \cdot 9 \\
12 \cdot .1 \\
11.9\end{array}$ & $\begin{array}{l}24 \cdot 6 \\
24 \cdot 7 \\
23 \cdot 5 \\
24 \cdot 2 \\
25 \cdot 2 \\
25 \cdot 2\end{array}$ \\
\hline 20 & $\begin{array}{l}\text { Father } \\
\text { Mother } \\
\text { Daughter } \\
\text { Son }\end{array}$ & $\begin{array}{l}49 \\
46 \\
20 \\
16\end{array}$ & $\begin{array}{l}\mathrm{R}+\mathrm{I} \cdot 66 \\
\mathrm{~L}+\mathrm{I} \cdot 97 \\
\mathrm{R}+\mathrm{I} \cdot 78 \\
\mathrm{~L}+\mathrm{I} \cdot 78 \\
\mathrm{R}-4.56 \\
\mathrm{~L}-3.83 \\
\mathrm{R}+\mathrm{I} \cdot 01 \\
\mathrm{~L}+\mathrm{I} \cdot 0 \mathrm{I}\end{array}$ & $\begin{array}{l}43 \cdot 5 \\
43.7 \\
45.3 \\
45.2 \\
44.4 \\
44.7 \\
43.0 \\
43 \cdot 2\end{array}$ & $\begin{array}{l}3.6 \\
3.5 \\
3.1 \\
2.9 \\
3.5 \\
3.5 \\
3.4 \\
3.5\end{array}$ & $\begin{array}{l}21 \cdot 3 \\
21 \cdot 9 \\
22 \cdot 6 \\
21 \cdot 9 \\
22 \cdot 5 \\
21 \cdot 3 \\
20 \cdot 9 \\
19 \cdot 4\end{array}$ & $\begin{array}{l}11 \cdot 7 \\
12 \cdot 1 \\
12 \cdot 7 \\
12 \cdot 6 \\
13 \cdot 7 \\
13 \cdot 1 \\
12 \cdot 3 \\
11 \cdot 5\end{array}$ & $\begin{array}{l}23.5 \\
23.1 \\
22.3 \\
22.4 \\
24.9 \\
24.7 \\
23.7 \\
23.9\end{array}$ \\
\hline
\end{tabular}




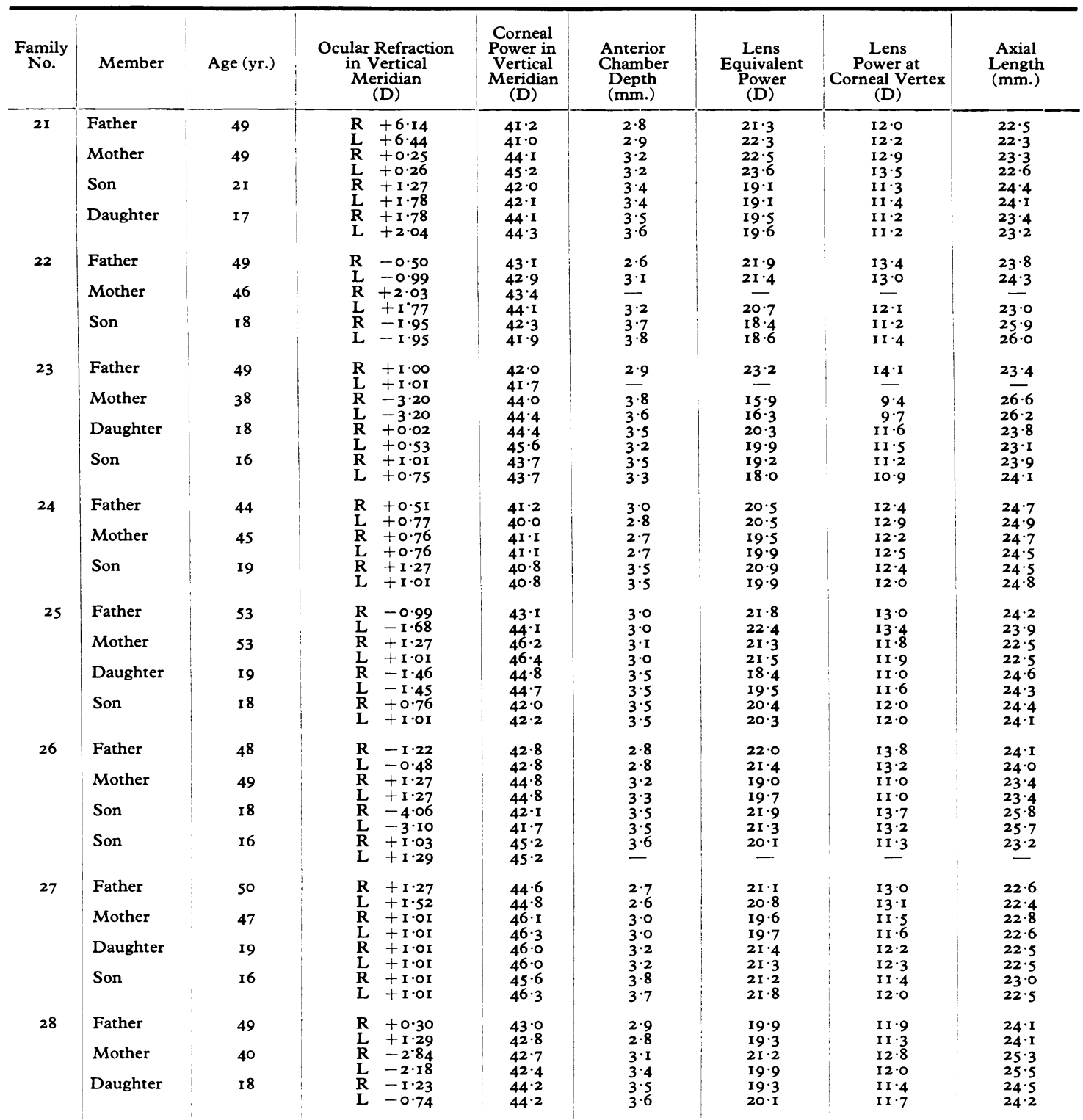

